\title{
Head Circumference as An Indicator of Nutritional Status in Critically III Children under 2 Years of Age
}

Alejandro Ochoa-Ramirez ${ }^{1}$, Evelyn M. Kuhn ${ }^{2}$, Jitsupa Sirinit ${ }^{3}$, Theresa A. Mikhailov ${ }^{2,3,}{ }^{*}$

1. University of lowa Hospitals and Clinics, 200 Hawkins Dr, lowa City, IA 52242, USA; E-Mail: alejandro-ochoaramirez@uiowa.edu

2. Children's Wisconsin, 8915 W Connell Ct, Milwaukee, WI 53226, USA; E-Mail: ekuhn@chw.org

3. Medical College of Wisconsin, 8701 W Watertown Plank Rd, Wauwatosa, WI 53226, USA; E-Mails: jsirinit@mcw.edu; tmikhail@mcw.edu

* Correspondence: Theresa A. Mikhailov; E-Mail: tmikhail@mcw.edu

Academic Editor: Gerhard Litscher

Special Issue: Artificial Nutrition in Pediatrics

OBM Integrative and Complementary Medicine Received: October 11, 2021

2022, volume 7 , issue 1

Accepted: January 17, 2022

doi:10.21926/obm.icm.2201003

Published: January 26, 2022

\begin{abstract}
Many clinical, biochemical, and anthropometric factors have been used to determine the nutritional status of critically ill children. One of the most commonly used anthropometric measurements in children is the head circumference. It is used to assess their development, and in some instances, can be an indicator of chronic malnutrition. We aim to study the association between head circumference and the nutritional status of critically ill children under 2 years old. We retrospectively obtained the head circumference measurements of 142 patients between 30 days and 2 years of age in the pediatric intensive care unit (PICU), and calculated their head circumference percentile-for-age based on CDC growth charts. We compared the head circumference percentiles to their nutritional status as determined by the Subjective Global Nutritional Assessment (SGNA) tool using the Mann-Whitney test. The median head circumference percentile was 9.5 (IQR 1.1-33.5) in patients with malnutrition and 54 (IQR 29.7-81.7) in patients with normal nutritional status $(P<0.001)$. There was no significant association between nutritional status and other possible confounders such as
\end{abstract}

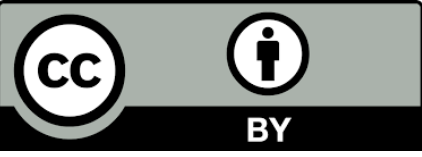

(C) 2022 by the author. This is an open access article distributed under the conditions of the Creative Commons by Attribution License, which permits unrestricted use, distribution, and reproduction in any medium or format, provided the original work is correctly cited. 
prematurity, age at admission, sex, and race by multivariate analysis. We found that critically ill patients with chronic malnutrition had significantly smaller head circumference than those with a normal nutritional status. However, it would be necessary to conduct a larger study in order to confidently determine a cutoff point for head circumference percentile that can be used to detect malnutrition in the PICU.

\section{Keywords}

Pediatric intensive care unit; PICU; pediatric critical care; pediatric malnutrition; head circumference

\section{Introduction}

Given the prevalence of malnutrition in the hospital setting, it is important to identify surrogate biochemical and clinical markers in order to quickly recognize and intervene [1]. Pediatric malnutrition is defined as "an imbalance between nutrient requirement and intake, resulting in cumulative deficits of energy, protein, or micronutrients that may negatively affect growth, development, and other relevant outcomes" and chronic malnutrition is defined as present for 3 months or longer [2]. For children, commonly used indicators of malnutrition include anthropometric markers such as BMI, weight-for-length, weight-for-age, height-for-age, and birth weight [3]. Other indicators include clinical markers such as weight loss, weight gain, or food intake [4]. Physical exam findings such as muscle wasting and adipose tissue distribution are also commonly used to determine nutritional status [5]. Another anthropometric parameter that is widely used in pediatrics is the head circumference [6]. However, it is more commonly used as a marker of development, while its use as a nutritional indicator has been limited.

Neurological development is highly dependent on the person's nutrition, particularly during the first 3 years of life $[7,8]$. This neurological development is reflected in the development of brain volume, of which head circumference is a reliable estimate [9-11]. Given the relationship between nutrition and head circumference, growth charts have been developed and are used in hospitals across the country [12].

One of the main limitations of using nationally representative growth charts is that the proportion of malnutrition in the general population is smaller than that of hospitalized patients. For example, common measures of malnutrition in children under five years of age such as stunting (low height-for-age) and wasting, (low weight-for-height) correspond to $3.5 \%$ and $0.4 \%$ respectively in the United States' population [13]. In contrast, the prevalence of malnutrition in the pediatric intensive care unit (PICU) has been found to be $24-53 \%$ in countries of various levels of economic development based on different anthropometric measurements [14]. Therefore, it is important to study the relationship between head circumference measurement and chronic malnutrition in the context of PICU admission.

Given the evidence presented, we hypothesize that critically ill patients that are chronically malnourished would have a smaller head circumference than those that have a normal nutritional status. Our specific aim is to determine if nutritional status affects head circumference in critically ill children under 2 years of age. A significant relationship between head circumference and 
nutritional status would support the use of this anthropometric parameter in the development of a nutrition screening tool in the future.

\section{Materials and Methods}

\subsection{Enrollment Criteria}

These patients had been previously enrolled in a larger, currently ongoing study to develop a nutrition screening tool. This is an ancillary study within that study. We retrospectively obtained the head circumference measurement of 142 patients under 2 years of age, which included children admitted to the pediatric intensive care unit (PICU) at Children's Wisconsin between 30 days and 18 years of age. Although CDC growth curves for head circumference include ages up to 3 years, our unit does not routinely record head circumference measurements for patients over 2 years of age.

Parents or legal guardians had already provided their informed consent to participate in the parent study. We received approval to conduct this ancillary study involving human subjects by the Children's Wisconsin institutional review board (IRB).

\subsection{Anthropometric Measurements}

We obtained anthropometric data from the electronic health record. These anthropometric measurements were determined by skilled pediatric critical care nurses in our unit at the time of admission to the PICU in accordance with national guidelines. The measurement of head circumference consists of measuring the patient's head with a nonelastic measuring tape from the occiput to the forehead just above the supraorbital ridge. Head circumference was recorded within 24 hours of admission. Patients with head circumference missing from the admission record were excluded from the study.

\subsection{Adjusting Head Circumference for Gestational Age}

We obtained the head circumference measurement in centimeters and the gestational age in weeks as recorded in the electronic health record. We entered the head circumference into a web calculator using the $2000 \mathrm{CDC}$ head circumference for age percentile [12]. We adjusted the head circumference percentile for gestational age at birth for any patient born before 40 weeks and then adjusted to 40 weeks' gestation.

\subsection{Determination of Nutritional Status}

We studied the statistical relationship between head circumference and other parameters that were obtained in the nutrition screen study. These parameters include prematurity, gestational age, demographic data, and nutritional status. The nutritional status was determined by a registered dietitian using the Subjective Global Nutritional Assessment (SGNA), which has been validated in the pediatric critical care population as a measure of nutritional status [15]. The SGNA takes into account a nutrition-focused medical history, which includes dietary intake, clinical symptoms, and weight changes, and a physical exam that measures subcutaneous fat, muscle wasting, and nutrition-related edema. The detailed methodology has been published for a precise reproduction of this assessment tool [5]. 


\subsection{Statistical Analysis}

We determined the association between head circumference and the nutritional status (based on the SGNA) using the Mann-Whitney test. We calculated the sensitivity and specificity for each head circumference percentile cut point between 0 and $100 \%$ for identification of malnutrition. Sensitivity was defined as the percentage of patients with malnutrition by SGNA who had a head circumference at or below the cut-off value, and specificity was the percentage of patients who were not malnourished by SGNA who had a head circumference above the cut-off value. Statistical analysis was performed using the R Statistical System [16] and IBM SPSS Statistics v20.

\section{Results}

A total of 142 patients were included in our study. From this cohort, 13 of them were malnourished (9.1\%) and 129 had normal nutrition status $(90.8 \%)$ as determined by SGNA. There was a greater representation of younger patients with more than $50 \%$ between 1 and 8 months of age (see Figure 1). However, there was no significant relationship between nutrition status and age. Other anthropometric and demographic data were compared to their nutrition status (see Tables 1 and 2). All the anthropometric parameters that we compared with nutrition status were significant, while the only demographic parameter that was significant was gestational age.

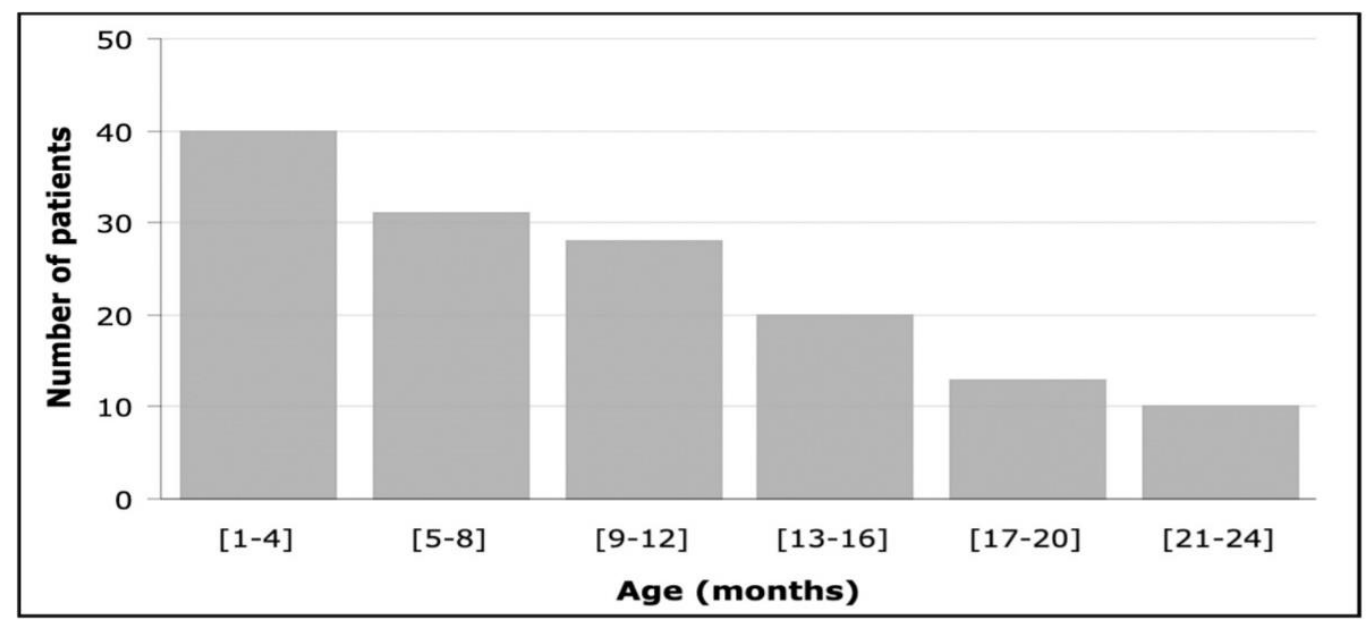

Figure 1 Age distribution of all participants. The frequency is shown for each age group of four contiguous months.

Table 1 Association between nutrition status and demographic parameters.

\begin{tabular}{llllll}
\hline & & \multicolumn{2}{l}{ Nutritional status } & $\begin{array}{l}\text { Total } \\
142\end{array}$ & $\begin{array}{l}\text { p- } \\
\text { value* }\end{array}$ \\
\cline { 3 - 5 } & $\begin{array}{l}\text { Malnourished } \\
(\mathrm{n}=13)\end{array}$ & $\begin{array}{l}\text { Normal } \\
(\mathrm{n}=129)\end{array}$ & & 0.563 \\
\hline Sex & Male & $9(69.2 \%)$ & $76(58.9 \%)$ & 85 & \\
& Female & $4(30.8 \%)$ & $53(41.1 \%)$ & 57 & 0.266 \\
Race & African American & $2(15.4 \%)$ & $25(19.4 \%)$ & 27 & \\
& Asian & $0(0.0 \%)$ & $2(1.6 \%)$ & 2 & \\
& Other & $0(0.0 \%)$ & $2(1.6 \%)$ & 2 & \\
\hline
\end{tabular}




\begin{tabular}{lllll}
\hline \multicolumn{1}{c}{ Caucasian } & $7(53.8 \%)$ & $89(69.0 \%)$ & 96 \\
$\quad$ Refused & $4(30.8 \%)$ & $11(8.5 \%)$ & 15 & \\
Prematurity & $5(38.5 \%)$ & $30(23.6 \%)$ & 35 & 0.311 \\
& Median (IQR) & Median (IQR) & & \\
\cline { 2 - 3 } Age at admission (months) & $5(3-11.5)$ & $9(4-14)$ & $8.5(4.0-14.0)$ & 0.135 \\
Gestational age at birth (weeks) & $37(35.3-38.0)$ & $38(36.0-39.0)$ & $38(36.0-39.0)$ & 0.037 \\
\hline
\end{tabular}

* Malnourished vs Normal nutritional status

Chi-square test for proportions and Mann-Whitney test for continuous numeric data.

Table 2 Association between nutrition status and anthropometric parameters.

\begin{tabular}{|c|c|c|c|c|c|c|c|}
\hline \multirow{3}{*}{ Measurements on admission } & \multicolumn{4}{|c|}{ Nutritional status } & \multirow{2}{*}{\multicolumn{2}{|c|}{ Total }} & \multirow{3}{*}{$p$-value* } \\
\hline & \multicolumn{2}{|c|}{ Malnourished } & \multicolumn{2}{|c|}{ Normal } & & & \\
\hline & $\mathrm{N}$ & Median (IQR) & $\mathrm{N}$ & Median (IQR) & $\mathrm{N}$ & Median (IQR) & \\
\hline Weight (kg) & 13 & $5.4(4.3-6.6)$ & 129 & $8.6(5.9-10.1)$ & 142 & $7.8(5.7-10.0)$ & $<0.001$ \\
\hline Length $(\mathrm{cm})$ & 13 & $64.0(56.2-66.0)$ & 127 & $71.0(62.0-76.2)$ & 140 & $68.4(61.3-76.0)$ & 0.006 \\
\hline Weight-for-age (percentile) & 13 & $0.2(0.01-0.44)$ & 128 & $40.5(12.6-70.1)$ & 141 & $34.9(7.7-68.4)$ & $<0.001$ \\
\hline Weight-for-height (percentile) & 13 & $2.0(0.24-12.9)$ & 120 & $53.5(15.7-83.6)$ & 133 & $39.0(12.0-81.8)$ & $<0.001$ \\
\hline
\end{tabular}

*Mann-Whitney test for Malnourished vs Normal status

We found a significant relationship between head circumference percentile-for-age and nutrition status. The head circumference percentiles were not normally distributed and had a range from 0 to 100 percentile in patients with normal nutrition status, and a range from 0 to 65.4 percentile in patients that were malnourished (see Figure 2).

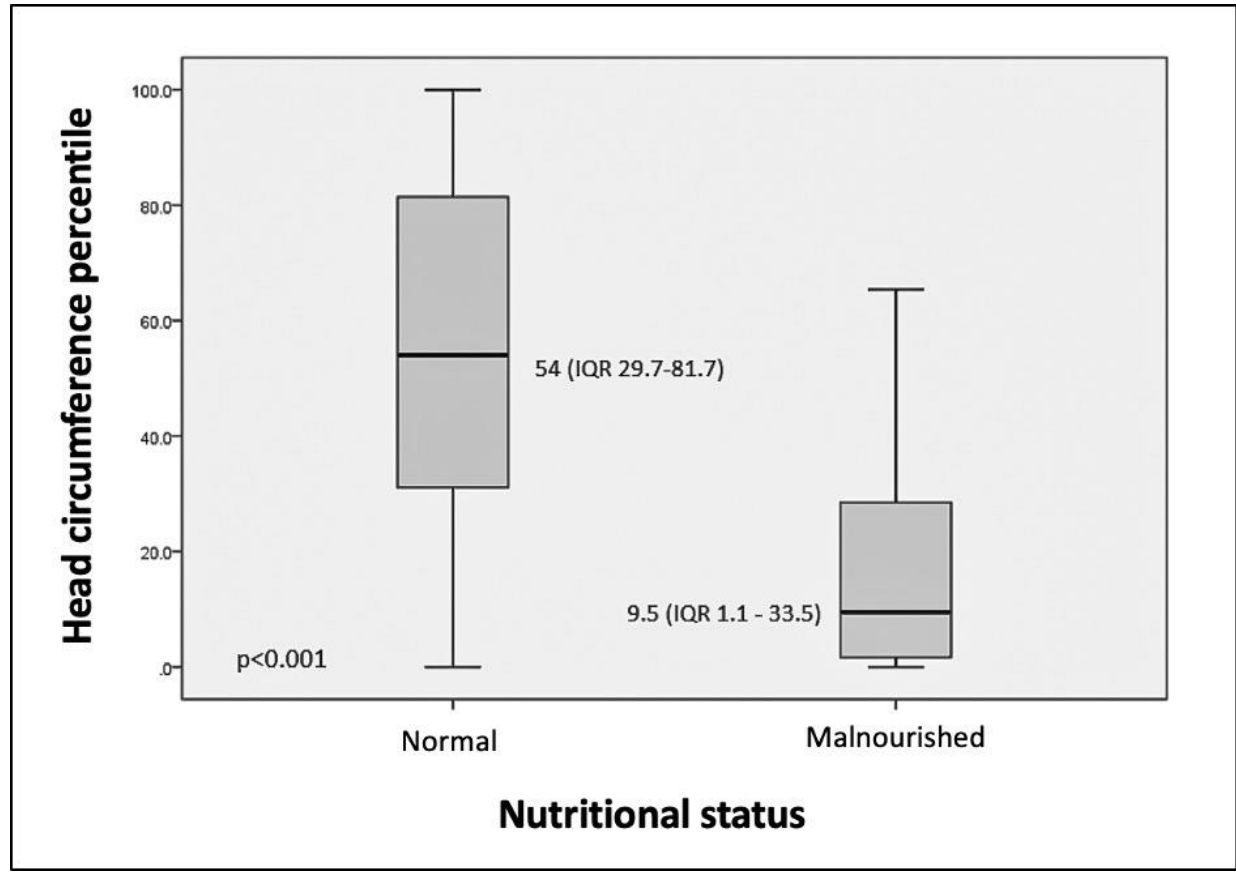

Figure 2 Head circumference percentile in normal and malnourished patients. The interquartile range is shown in the box with a solid line at the median head circumference value. 
We plotted a receiver operating characteristic $(R O C)$ curve showing sensitivity and specificity for all head circumference percentiles (see Figure 3 ). The intersection occurs at a head circumference percentile close to 29 with a sensitivity of 76.92 and a specificity of 75.19.

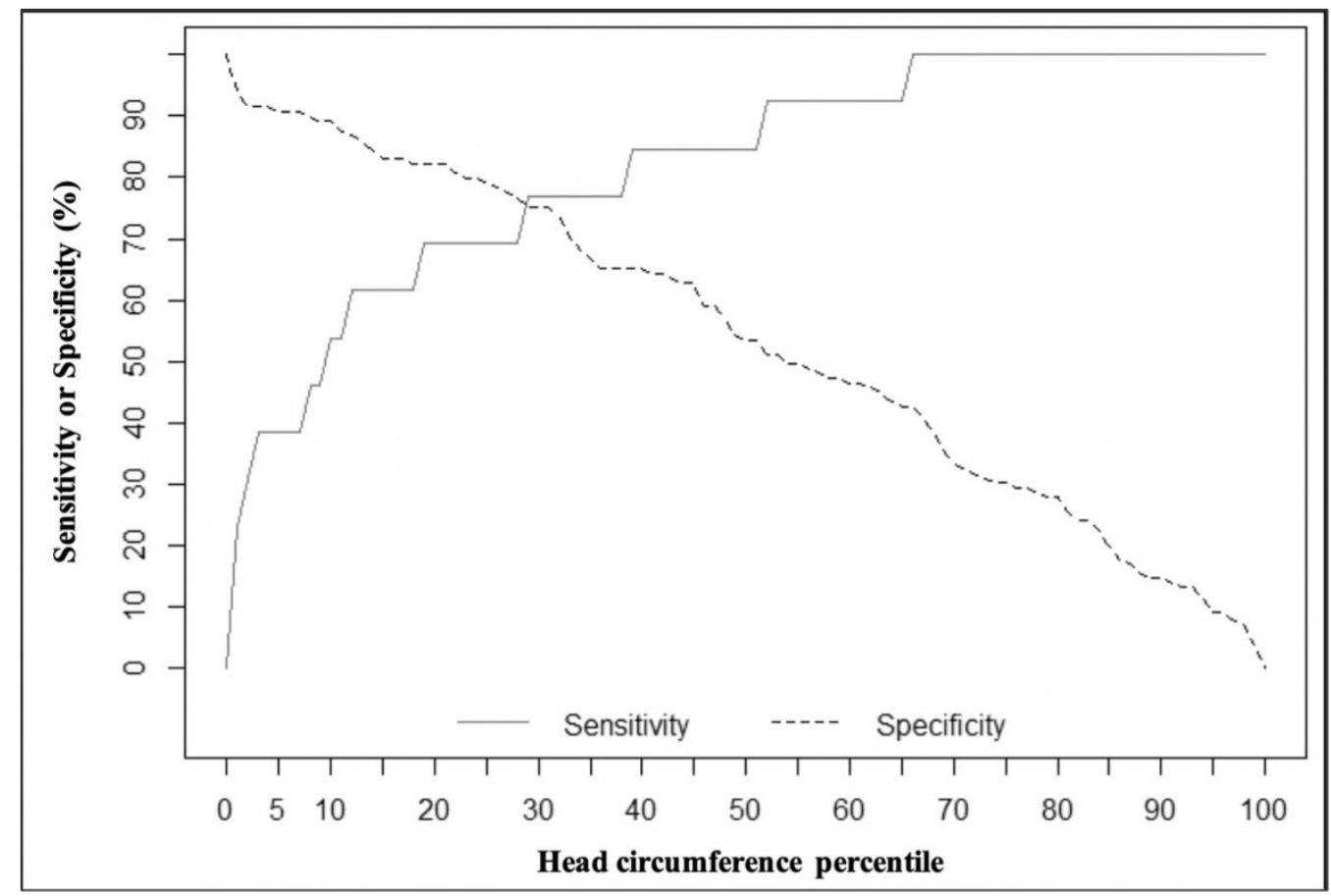

Figure 3 Sensitivity and specificity by head circumference percentile. Values for sensitivity and specificity at all head circumference percentile values between 0 and 100 .

Additionally, we analyzed the relationship between head circumference percentile and prematurity, but the difference was not significant with a $P$ value of 0.311 . While prematurity itself was not a significant factor, the gestational age was less in malnourished patients [37 (35.3-38.0) median (IQR) in weeks] than in patients with normal nutrition status [38 (36.0-39.0) median (IQR) in weeks]. A multivariate logistic regression analysis that included prematurity, age at admission, male sex, African American and Caucasian race, and head circumference percentile showed no significant associations except for head circumference percentile, consistent with the findings from the univariate analysis.

\section{Discussion}

As we hypothesized, patients that were malnourished had a statistically significant smaller head circumference than the patients that had a normal nutritional status. It is important to notice that our head circumference measurements were adjusted for gestational age and when comparing the head circumference percentile of premature patients with term patients, no significant difference was found. From our univariate and multivariate analyses, we did not find prematurity to be a determining factor of nutritional status or head circumference percentile in our cohort. As previously noted, there was a significant difference between the gestational ages at birth in patients that were malnourished and patients with normal nutrition status, with a younger gestational age in patients that were malnourished. 
Given the significant association, it is possible to use head circumference to identify chronic malnutrition in critically ill children. A screening tool that detects malnutrition allows us to prioritize these patients for early nutritional intervention. Therefore, it is preferred to have a screening tool with high sensitivity because it is ultimately better to treat falsely detected malnourished patients than delay intervention when a malnourished patient is missed. Using only our study population, an ideal cutoff point for head circumference percentile would be between 30 and 35, given that at this range, sensitivity is greater than specificity, and it is below the interquartile range for patients with normal nutritional status.

One of the major limitations of our study is the small sample size. Although we obtained statistically significant results, these might only be representative of our cohort. Other limitations are related to the retrospective nature of our study. These include the possibility of increased confounding factors, which were addressed by multivariate analysis, and the inability to establish causation, although the association that we found should be sufficient for the purpose of creating a screening tool.

Another limitation of our study is the wide variety of diagnoses, particularly chronic illnesses that are present in the pediatric intensive care unit population. Some of these are a direct cause of malnutrition, which would result in a smaller head circumference. Some other chronic illnesses, however, may result in a smaller head circumference despite normal nutrition and could contribute to a decreased accuracy in our measurements.

There are other anthropometric parameters, such as weight for height and mid-upper arm circumference, that are commonly used as part of the WHO standards [17] to assess for malnutrition. However, these tools are for children up to 5 years of age and they are focused on acute malnutrition, with very limited studies on chronic malnutrition. Furthermore, recent studies have shown that coupling these measurements together failed to recognize severe confounding factors such as edema [18]. Our study adds another data point for the development of a multifactorial screening tool that is also more specific to critically ill children under 2 years of age.

In this study we elucidated a significant difference between the head circumference of patients with chronic malnutrition and patients with normal nutrition status. With this foundation, the next step would be to validate our findings in a larger, prospective study in order to confidently determine a cutoff point that can be used to screen patients for malnutrition in the PICU. Head circumference percentile should also be used in conjunction with other significant anthropometric and clinical parameters to create a more effective nutrition screening tool.

\section{Author Contributions}

Alejandro Ochoa-Ramirez designed the study, collected data, wrote the first draft of the manuscript, and critically revised the manuscript. Evelyn M. Kuhn performed the data analysis, interpreted analysis of the data, and critically revised the manuscript. Jitsupa Sirinit collected data and critically revised the manuscript. Theresa A. Mikhailov designed the study, interpreted analysis of the data, and critically revised the manuscript. All authors reviewed and approved the final version of the manuscript.

\section{Competing Interests}

None declared. 


\section{References}

1. Seres DS. Surrogate nutrition markers, malnutrition, and adequacy of nutrition support. Nutr Clin Pract. 2005; 20: 308-313.

2. Mehta NM, Corkins MR, Lyman B, Malone A, Goday PS, Carney L, et al. Defining pediatric malnutrition: A paradigm shift toward etiology-related definitions. J Parenter Enteral Nutr. 2013; 37: 460-481.

3. WHO. Physical status: The use and interpretation of anthropometry. Report of a WHO Expert Committee. Technical Report Series No. 854. Geneva: World Health Organization; 1995.

4. White M, Lawson K, Ramsey R, Dennis N, Hutchinson Z, Soh XY, et al. Simple nutrition screening tool for pediatric inpatients. J Parenter Enteral Nutr. 2016; 40: 392-398.

5. Secker DJ, Jeejeebhoy KN. How to perform subjective global nutritional assessment in children. J Acad Nutr Diet. 2012; 112: 424-431.e6.

6. Martini M, Klausing A, Lüchters G, Heim N, Messing-jünger M. Head circumference - a useful single parameter for skull volume development in cranial growth analysis? Head Face Med. 2018; 14: 1-8.

7. Schwarzenberg SJ, Georgieff MK, Committee on Nutrition, Daniels S, Corkins M, Golden NH, et al. Advocacy for improving nutrition in the first 1000 days to support childhood development and adult health. Pediatrics. 2018; 141: e20173716.

8. Fox SE, Levitt $P$, Nelson CA. How the timing and quality of early experiences influence the development of brain architecture. Child Dev. 2010; 81: 28-40.

9. Bartholomeusz $\mathrm{HH}$, Courchesne E, Karns CM. Relationship between head circumference and brain volume in healthy normal toddlers, children, and adults. Neuropediatrics. 2002; 33: 239241.

10. Cooke RW, Lucas A, Yudkin PL, Pryse-Davies J. Head circumference as an index of brain weight in the fetus and newborn. Early Hum Dev. 1977; 1: 145-149.

11. Prado EL, Dewey KG. Nutrition and brain development in early life. Nutr Rev. 2014; 72: 267284.

12. CDC. Growth charts - 2000 CDC growth charts - United States [Internet]. US: CDC; 2020 [cited 2020 October 26]. Available from: https://www.cdc.gov/growthcharts/cdc charts.htm.

13. Global Nutrition Report. United States of America nutrition profile - Global nutrition report [Internet]. US: Global Nutrition Report; 2020 [cited 2020 October 29]. Available from: https://globalnutritionreport.org/resources/nutrition-profiles/north-america/northernamerica/united-states-america/.

14. Skillman HE, Mehta NM. Nutrition therapy in the critically ill child. Curr Opin Crit Care. 2012; 18: 192-198.

15. Vermilyea S, Slicker J, El-Chammas K, Sultan M, Dasgupta M, Hoffmann RG, et al. Subjective global nutritional assessment in critically ill children. J Parenter Enteral Nutr. 2013; 37: 659-666.

16. R Core Team. R: A language and environment for statistical computing. R Foundation for Statistical Computing, Vienna, Austria. 2021. Available from: http://www.R-project.org/.

17. WHO. The WHO child growth standards [Internet]. Geneva: WHO; 2006. Available from: https://www.who.int/tools/child-growth-standards. 
18. Grellety E, Golden MH. Severely malnourished children with a low weight-for-height have a higher mortality than those with a low mid-upper-arm-circumference: I. Empirical data demonstrates Simpson's paradox. Nutr J. 2018; 17: 1-21.

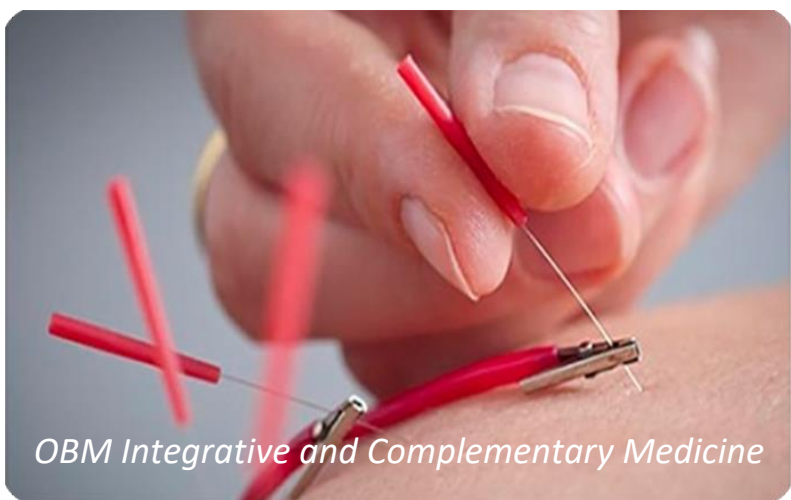

Enjoy OBM Integrative and Complementary Medicine by:

1. Submitting a manuscript

2. Joining in volunteer reviewer bank

3. Joining Editorial Board

4. Guest editing a special issue

For more details, please visit:

http://www.lidsen.com/journals/icm 